\title{
BMJ Open Innovative Telemonitoring Enhanced Care Programme for Chronic Heart Failure (ITEC-CHF) to improve guideline compliance and collaborative care: protocol of a multicentre randomised controlled trial
}

\author{
Hang Ding, ${ }^{1}$ Rajiv Jayasena, ${ }^{2}$ Andrew Maiorana, ${ }^{3,4}$ Alison Dowling, ${ }^{5}$ \\ Sheau Huey Chen, ${ }^{4}$ Mohan Karunanithi, ${ }^{1}$ Jamie Layland, ${ }^{6}$ Iain Edwards ${ }^{5}$
}

To cite: Ding $\mathrm{H}$, Jayasena $\mathrm{R}$ Maiorana A, et al. Innovative Telemonitoring Enhanced Care Programme for Chronic Heart Failure (ITEC-CHF) to improve guideline compliance and collaborative care: protocol of a multicentre randomised controlled trial. BMJ Open 2017;7:e017550. doi:10.1136/ bmjopen-2017-017550

- Prepublication history and additional material for this paper are available online. To view please visit the journal (http:// dx.doi.org/10.1136/bmjopen2017-017550).

Received 2 May 2017 Revised 28 July 2017 Accepted 6 September 2017

CrossMark

For numbered affiliations see end of article.

Correspondence to

Dr Hang Ding;

hang.ding@csiro.au

\section{ABSTRACT}

Introduction Chronic heart failure (CHF) is a lifethreatening chronic disease characterised by periodic exacerbations and recurrent hospitalisations. In the management of $\mathrm{CHF}$, patient compliance with evidencebased clinical guidelines is essential, but remains difficult practically. The objective of this study is to examine whether an Innovative Telemonitoring Enhanced Care Programme for CHF (ITEC-CHF) improves patients' compliance, and associated health and economic outcomes.

Methods and analysis An open multicentre randomised controlled trial has been designed. Patients will be recruited and randomised to receive either ITEC-CHF $(n=150)$ or usual care CHF $(n=150)$ for at least 6 months. ITEC-CHF combines usual care and an additional telemonitoring service including remote weight monitoring, structured telephone support and nurse-led collaborative care. The primary outcomes are the compliance rates with the best-practice guidelines for daily weight monitoring.

The secondary outcomes include the compliance with other guideline recommendations (health maintenance, medication, diet and exercise), health (health-related quality of life, risk factors, functional capacity and psychological states) and economic outcomes related to the use of healthcare resources such as hospital readmissions and general practitioner/emergency department visits.

Ethics and dissemination The clinical trial has been approved by Peninsula Health Human Research Ethics Committee (HREC Reference: HREC/14/PH/27), Royal Perth Hospital Human Research Ethics Committee (Reference: 15-081) and the Curtin University Human Research Ethics Committee (Reference: HR 181/2014). We will disseminate the final results to the public via conferences and journal publications. A final study report will also be provided to the ethics committees.

Trial registration number Registered with Australian New Zealand Clinical Trial Registry (ACTRN12614000916640).
Strengths and limitations of this study

- A multicentre randomised controlled trial to evaluate the effects of telemonitoring on patient compliance with guideline-advocated self-management, and associated clinical outcomes.

- Special focus on addressing the well-known issue of patient non-compliance with daily weight management.

- Novel 'zero-touch' design to prevent any technical difficulties and weight monitoring burdens to participants.

- Integration with the best-practice clinical workflows and action plan to streamline the intervention processes and minimise the workload to the care providers.

- The study is limited to a 6-month intervention, potentially insufficient for analysis of long-term effects on patient compliance and health outcomes.

\section{INTRODUCTION}

Congestive heart failure (CHF), a life-threatening chronic disease, is a global pandemic. ${ }^{1}$ It affects over 26 million people ${ }^{2}$ with annual direct costs of over US $\$ 65$ billion globally. ${ }^{3}$ Despite advances of modern medicine, patients with CHF continue to experience poor survival $\left(30 \%-50 \%\right.$ at 5 years $\left.{ }^{45}\right)$, debilitating symptoms such as dyspnoea and fatigue, ${ }^{6}$ poor health-related quality of life $(\mathrm{HQOL})^{7}$ and episodic clinical exacerbations with high risks of hospitalisation. ${ }^{89}$

To effectively manage CHF, evidence-based guidelines are available internationally. ${ }^{10} \mathrm{~A}$ consistent recommendation of guidelines is self-management, such as fluid restrictions and daily weighing to monitor fluid balance. ${ }^{12}$ Patient compliance with such clinical 
guidelines is essential to optimise health outcomes, ${ }^{13}$ but is often suboptimal. ${ }^{14}{ }^{15}$ For example, $12 \%-75 \%$ of patients in usual care were found to adhere to the cardinal recommendation of daily weighing. ${ }^{15}{ }^{16}$ Non-compliance is likely due to a variety of factors including time constraints ${ }^{17}$, insufficient knowledge ${ }^{18}$ and limited clinical support. ${ }^{19}$ Importantly, patients who are non-compliant with self-management guidelines often fail to effectively engage with clinicians for timely interventions, ${ }^{16}$ resulting in increased risks of mortality ${ }^{20}$ and hospital readmissions. ${ }^{21} 22$

Telemonitoring applications are an evolving strategy to improve CHF care, which have the potential to support patients in self-management. ${ }^{23-25}$ However, the effect of telemonitoring on patient compliance with guideline-advocated self-management has not been extensively studied. To our knowledge, only four randomised controlled trials (RCTs) have compared patient compliance between a telemonitoring intervention and usual care group. ${ }^{26-29}$ These trials were limited by small sample sizes $(n \leq 100)$ or short-duration follow-up ( $\leq 3$ months). Furthermore, their outcomes are inconsistent, with two trials reporting improved patient compliance ${ }^{2627}$ and two reporting no benefit. ${ }^{28} 29$ Because of the limited and inconsistent evidence, no meta-analysis studies or reviews have been able to conclude the effectiveness of using telemonitoring to improve patient compliance. These facts highlight the need for further research to substantiate patient compliance in telemonitoring studies for $\mathrm{CHF}$ care.

To address the issue of patient non-compliance, we have designed an Innovative Telemonitoring Enhanced Care Programme for CHF (ITEC-CHF). This programme focuses on assisting patients with $\mathrm{CHF}$ in complying with the daily weight management recommended by the guidelines. To minimise weight monitoring burdens and technical difficulties, the programme proposes a novel 'zero-touch' design, meaning that the participants are not required to interact with the technology other than stepping onto a scale for weight measurement as in usual care, and they do not need to learn extra knowledge and skills to receive the telemonitoring intervention. The programme is also integrated with existing best-practice clinical workflows and action plan to streamline the intervention and make it seamless for care providers.

To evaluate the programme, we will conduct a multicentre RCT. The objective of the trial is to examine the hypothesis that the ITEC-CHF improves patients' compliance, and associated health and economic outcomes. This trial will examine the patient compliance of an innovative telemonitoring programme across different care settings. It will also add essential clinical evidence to support use of telemonitoring applications in the community.

\section{METHODS}

\section{Trial design}

A prospective open two-arm multicentre RCT has been designed. In the trial, patients with CHF will be recruited at two trial sites: (1) Frankston Hospital and Rosebud Hospital in Victoria (VIC), Australia, and (2) Royal Perth Hospital and Fiona Stanley Hospital in Western Australia (WA).

The participants in the trial will be individually randomised to receive either ITEC-CHF or usual care (UC-CHF) for at least 6 months. The allocation ratio of the randomisation is $1: 1$. The randomisation is stratified by the two trial sites (VIC and WA) to ensure the allocation ratio in each site. According to a recommended method ${ }^{30}$, a series of random allocation assignments with permuted blocks have been generated and sealed in opaque envelopes by two research scientists in a research organisation in the study. The block sizes will be kept confidential to prevent potential prediction of the assignments. Data analysts generated the randomisation sequence, and will be blinded throughout the trial using de-identified patient data. It will be difficult to effectively blind the participants and care providers to telemonitoring interventions, but we will attempt to avoid unnecessary discussions about the allocations and hypothesised outcomes with the participants and care providers throughout the trial.

The trial flow diagram is presented in figure 1. Through the trial, project nurses at the trial sites will use their electronic patient administration systems to screen patients with CHF from medical records of patient presentations at the local hospitals and emergency departments (EDs). They will also screen patient records at heart failure outpatient clinics, and community healthcare services. The nurses will then record eligible candidates, and accordingly send an invitation letter to the candidates. One week later, project nurses will follow up with the candidates via a telephone call. If a candidate is willing to participate in the trial, the project nurses will arrange a face-to-face interview. During the interview, project nurses will explain the trial processes and requirements in detail, and conduct formal written consent with the candidates who agree to participate in the trial. Participants recruited will be interviewed by the project nurses for a baseline assessment. Each participant will then be randomised into either ITEC-CHF or UC-CHF group. At the 6-month time point, all participants will be assessed again. After the 6-month assessment, if the participants are willing to continue, they can stay in the trial to receive a 12-month assessment. Finally, the project nurses will collect the trial data for the research analysis. Participants will be enrolled from January 2015 to October 2017.

\section{Inclusion and exclusion criteria}

Patients will be eligible to participate in the trial, if they satisfy all the following inclusion criteria: (1) CHF diagnosed by a clinician with an ejection fraction $\leq 40 \%$, (2) able to weigh themselves safely, (3) at least 18 years of age, 


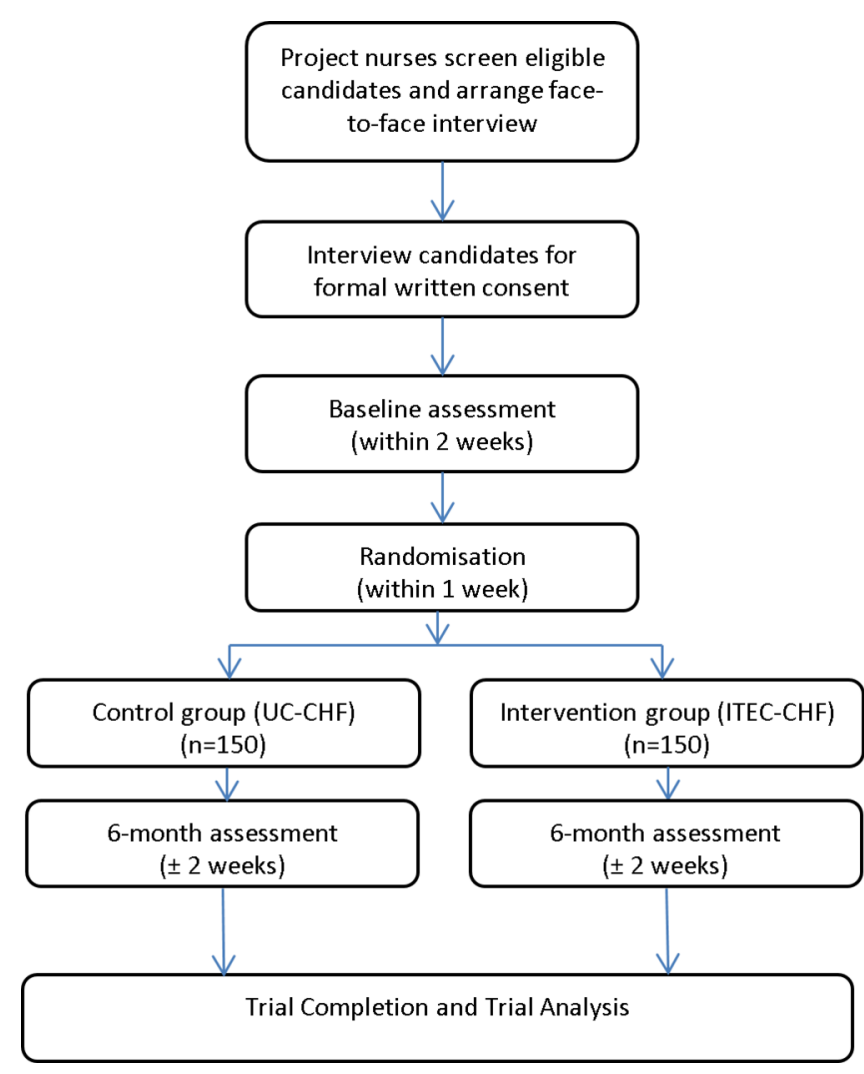

Figure 1 Trial flow diagram of the two-arm randomised controlled trail to compare the Innovative Telemonitoring Enhanced Care Programme for Congestive Heart Failure (ITEC-CHF) with usual care CHF (UC-CHF).

(4) have a regular personal general practitioner (GP) or agree to use a designated GP, (5) with a permanent residential address and (6) without significant cognitive impairments. Patients will be excluded if they meet any of the following criteria: (1) expected survival $<12$ months, such as patients with documented palliative care in medical records, (2) end-stage renal failure on dialysis, (3) long-term nursing home resident or (4) participating in any other clinical trial.

\section{Trial interventions}

\section{Usual care}

Participants in the UC-CHF group will receive a standard package of paper-based diary and booklets at baseline, including the 'Living Well with Chronic Heart Failure' resource produced by the Heart Foundation of Australia (HFA). They will then attend standard CHF clinics and primary care physicians and undertake traditional CHF self-management through the trial period. Each participant will also be provided with an electronic weighing scale (ForaCare, W550, Moorpark, CA, USA), and asked to use the weight scale according to the self-management recommendation of the HFA. Project nurses will visit the participants to download the weight data from the weight scale at scheduled times, approximately every 3 months.

\section{Enhanced care}

The ITEC-CHF will combine usual care and an additional telemonitoring service. The telemonitoring service consists of three major components: remote weight monitoring, structured telephone support and nurse-led collaborative care. The service is integrated with a telephone call centre (MePACS, VIC, Australia) ${ }^{31}$ and a nurse care service according to their workflows in usual care.

The integrated care model of the ITEC-CHF is shown in figure 2. In the model, participants are provided with an electronic weighing scale (ForaCare, W550), and a computer tablet (Samsung, Galaxy Tab A, Seoul, Korea). They are asked to use the scale to measure their body weight daily, immediately after wake-up, following voiding, without shoes, in light clothing and before the next dose of medication. The measured weight entry is recorded in the scale, and then automatically transmitted to the tablet via a wireless Bluetooth function embedded in the scale. The tablet is preloaded with an Android application (Medtech Global, Melbourne, Australia). This application receives the weight entry, and uploads the entry to a proprietary software package, called ManageMyHealth (MMH) (Medtech Global). A web application in $\mathrm{MMH}$ automatically monitors uploaded weight entries in real time to generate alerts, and triage the alerts to project nurses and the telephone call centre. The rules to generate and triage the alerts are given in table 1 . They were designed in accordance with the HFA guidelines for the prevention, detection and management of CHF in Australia. ${ }^{10}$

Operators at the call centre will respond to the alerts in real time (24hours, 7 days a week). If a participant does not weigh before $10 \mathrm{AM}$, a call operator will call the participant to remind him/her to weigh. During the call, if the participant needs clinical support such as advice for assessing CHF symptoms or managing diet, the call operator will arrange a nurse follow-up. The project nurses will subsequently follow up with the participant via a telephone call or home visit if needed. The call operators also provide technical support through the trial, such as updating the tablet application, and arranging home visits to change batteries in the scales.

The project nurses provide structured interventions according to three types of alerts: rapid weight fluctuation ( $\pm 2 \mathrm{~kg}$ in 2 days), slow weight fluctuation $( \pm 5 \mathrm{~kg}$ in 28 days) and low-risk weight fluctuation $( \pm 1 \mathrm{~kg}$ over 24 hours). If a participant has rapid weight fluctuation, a project nurse will be alerted. The nurse will then call the participant to assist him/her in assessing critical symptoms, and activating the CHF action plan if indicated, such as attending their GP, CHF clinic or presenting to an ED. For an alert of slow weight fluctuation, the project nurses will assist the participant in assessing CHF symptoms, and arranging clinical review at the participants' GP or CHF clinics. The option to present to an ED may also be applied, if this is clinically indicated. If a participant's body weight fluctuation exceeds $\pm 1 \mathrm{~kg}$ (but is less than $\pm 2 \mathrm{~kg}$ ) over 24 hours, a 


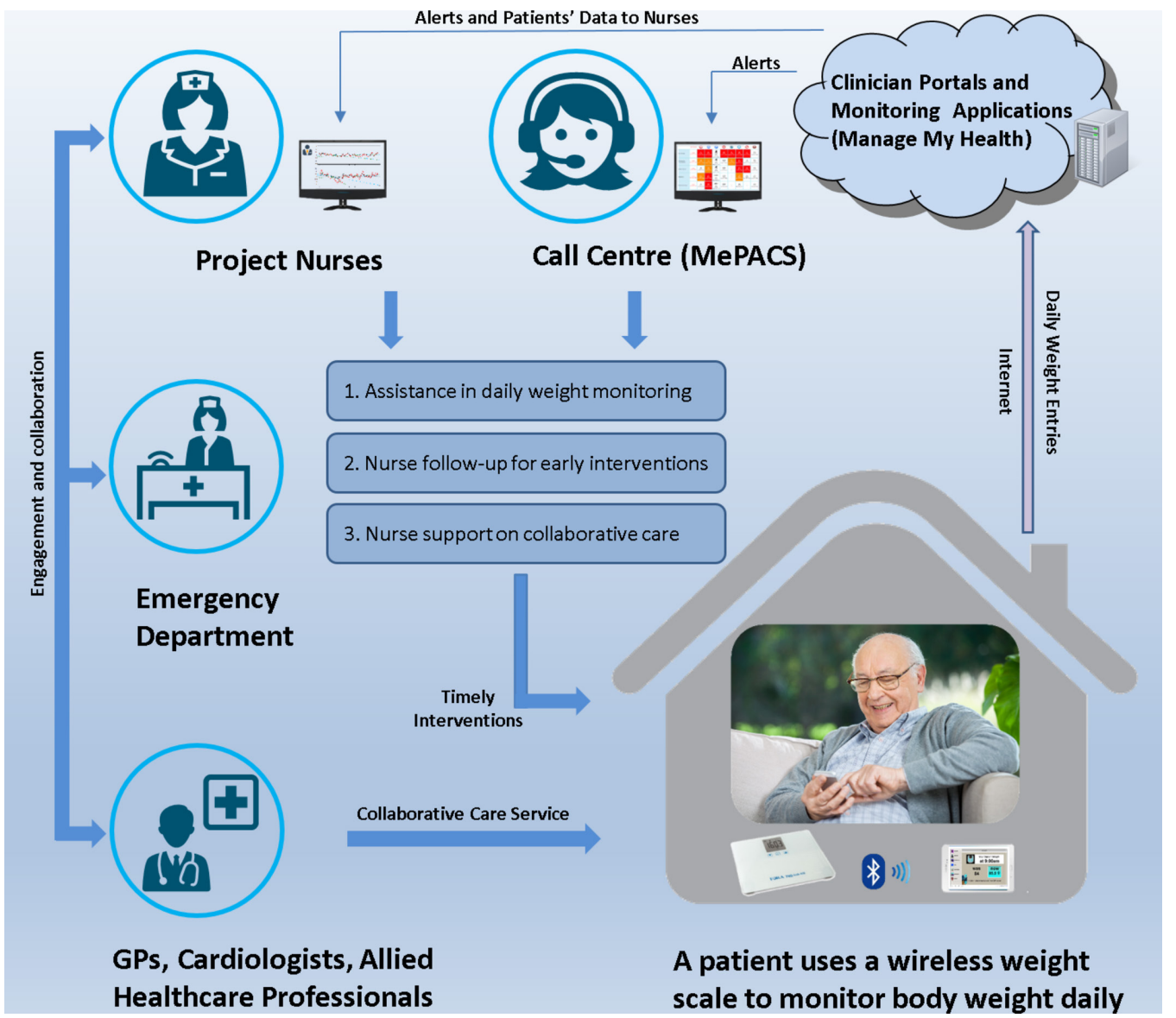

Figure 2 The care model of the Innovative Telemonitoring Enhanced Care Programme for Congestive Heart Failure is integrated within usual care. The integration includes: (1) remote weight monitoring, (2) structured telephone support and (3) nurse-led collaborative care. GP, general practitioner.

questionnaire will be automatically triggered and sent to the participant's computer tablet. The user interface of the questionnaire is shown in online Supplementary appendix 1 . If the participant reports any of the clinical conditions in the questionnaire or does not respond to the questionnaire, the project nurses will follow up with the participant for a clinical assessment.

\section{Primary and secondary outcomes}

The outcome measures of the trial are given in table 2 . The primary outcome will be the patient compliance with daily weight monitoring as evaluated by weight entries, recorded on the electronic scales provided to both the ITEC-CHF and UC-CHF groups. Based on a published

Table 1 Alerts generated, and associated interventions provided in the Innovative Telemonitoring Enhanced Care Programme for Congestive Heart Failure (CHF)

\begin{tabular}{|c|c|c|c|}
\hline Care provider & Conditions & Response time & Interventions \\
\hline $\begin{array}{l}\text { Call centre } \\
\text { (MePACS) }\end{array}$ & $\begin{array}{l}\text { No weight entry detected } \\
\text { before } 10 \mathrm{AM} \text {. }\end{array}$ & $\begin{array}{l}\text { In real time, } 24 \text { hours, } \\
7 \text { days a week. }\end{array}$ & $\begin{array}{l}\text { Operators at MePACS call the patients to remind them } \\
\text { to weigh. If needed, they call nurses to follow up. }\end{array}$ \\
\hline \multirow[t]{2}{*}{ Project nurses } & Fluctuation of $2 \mathrm{~kg}$ in 2 days. & $\begin{array}{l}\text { In real time, } 24 \text { hours, } \\
7 \text { days a week. }\end{array}$ & $\begin{array}{l}\text { Nurses call patients for further assessment and help } \\
\text { process the CHF action plan. }\end{array}$ \\
\hline & $\begin{array}{l}\text { Fluctuation of } 1 \mathrm{~kg} \text { over } \\
24 \text { hours. }\end{array}$ & $\begin{array}{l}\text { In real time, } 24 \text { hours, } \\
7 \text { days a week. }\end{array}$ & $\begin{array}{l}\text { A questionnaire will be automatically triggered on the } \\
\text { table to assess clinical symptoms. If the participant } \\
\text { has any of the symptoms assessed, the nurses will be } \\
\text { notified to follow up with the patient for intervention. }\end{array}$ \\
\hline
\end{tabular}


Table 2 Trial outcome measures and assessment tools and data resources

Primary outcome

Compliance with daily weight monitoring

Daily weight entries recorded in participant's scale and $\mathrm{MMH}$

Secondary outcomes

Other guideline recommendations to the $\mathrm{CHF}$ management

Heart Failure Compliance Questionnaire (health maintenance,

Health/clinical outcomes medication, diet and exercise)

Variation of body weight (mean and SD of weekly weight entries)

Functional capacity (6 min walk test)

Health-related quality of life (EQ-5D)

Psychosocial status (CDS, short version)

Frailty scale (Canadian Frailty Index)

Health economic outcomes

Number of hospital readmissions, and length of stay (mean, SD)

Number of ED visits, and length of stay (mean, SD)

Number of GP visits

CHF, congestive heart failure; ED, emergency department; GP, general practitioner; MMH, Manage My Health.

study, ${ }^{14}$ we define that a participant is compliant with daily weight monitoring, if he/she performed weight monitoring at least 4 days a week. The rate of compliant days with weight monitoring (days with at least one body weight entry) per week for each participant during his/ her trial period will also be computed, and the histogram of the rate will be analysed. The secondary outcomes will include compliance with other guideline recommendations assessed by Heart Failure Compliance Questionnaire $^{32}$ (health maintenance, medication, diet and exercise), health outcomes including HQoL (European Quality of Life Five Dimension, EQ-5 $\left.\mathrm{D}^{33}\right)$, risk factors, functional capacity (6 min walk test ${ }^{34}$ ), psychological states (Cardiac Depression Scale, CDS-SF2 ${ }^{35}$ ) and frailty scale (Canadian Frailty Index ${ }^{36}$ ), and health economic outcomes related to the use of healthcare resources such as hospital readmissions and GP/ED visits. Patients' characteristics and assessment records will be collected by the project nurses. For evaluation of hospital readmissions and ED presentations, the data will be extracted from electronic patient administration systems of the hospitals in the trial.

\section{Strategies of participant retention}

Prior to the trial recruitment, we will discuss the importance of participant retention within the recruitment and care teams. During the trial, each participant recruited will be provided with a telephone contact in the information package. Through the contact, the participants can contact project nurses for trial-related support. A structured procedure will be in place to guide project nurses to document the participants' enquiries, and ensure timely responses and/or follow-ups. Moreover, the project nurses will visit each participant every 3 months, to discuss any concerns or issues related to the trial and change the batteries in the weight scale.

\section{Participant discharge}

Participants will be discharged from the trial under the conditions of (1) mortality, (2) elective withdrawal and (3) full completion of the programme. When discharging participants, projects nurses will document the conditions in case report forms (CRF) with date and causes in detail.

\section{Data security and storage}

All trial files, including the master list, CRF and clinical assessment forms, will be stored securely, either in password-protected computer files or in locked filing cabinets in a secure area at Peninsula Health or Curtin University. Access to these files will only be granted to study personnel trained in confidentiality and privacy procedures at the hospitals in the study. All trial data provided for research analysis will be de-identified, including patient characteristics, primary and secondary outcomes and data entries through the healthcare and telemonitoring systems in the study.

All the trial files and data will be stored securely for a minimum of 5 years after completion of the study and, finally, be securely destroyed according to Privacy Policy provided by the National Health and Medical Research Council of Australia.

\section{Sample size}

Compliance with daily weight monitoring was used to calculate the sample size. The null hypothesis is that the percentage of 'compliant' participants in the ITEC-CHF group is not higher than in the UC-CHF group. To reject the null hypothesis, we assumed a compliance rate of $80 \%$ in ITEC-CHF, and 65\% in UC-CHF. We also assumed an attrition rate of $10 \%$. A two-tailed test with a power of $90 \%$ and alpha of 0.05 was used to calculate the same size to achieve a statistical significance ${ }^{37}$. The power calculation resulted in that the study needs at least 143 participants in each group. Accordingly, we rounded the calculated 
sample size, and made the trial size of 150 participants in each group, for a total of 300 participants. With this sample size, we have a likelihood of $90 \%$ to yield a statistical significance.

\section{Statistical analysis}

All participants recruited and randomised into the two trial groups will be included in the final comparative analysis according to the intention-to-treat design. A $\chi^{2}$ test will be used to compare categorical variables between the ITEC-CHF and UC-CHF group, such as sex, and compliance (compliant patients vs non-compliant patients). Analysis of variance will be applied to analyse continuous variables such as age and weight variations. A Cox proportional hazards model will be performed to analyse the risks of hospital readmission and ED visits. The analysis will be adjusted for confounding variables including sex, age and trial sites. The CI of $95 \%$ will be estimated. A $\mathrm{p}$ value of less than 0.05 will be considered as statistical significance for all tests. The statistical analysis will be conducted using SPSS V.23. Missing data at the case level will be imputed using a multiple imputation method in the SPSS.

\section{Trial management}

A Project Working Group (PWG) will be composed of the chief investigators, research scientists or project managers from the organisations of the project. The PWG will convene monthly (with additional meetings if needed) and take overall responsibility for the conduct of the trial, such as managing the trial progress, reviewing adverse events in CRF, resolving technical issues and monitoring trial data. If necessary, the committee will advise and make changes in the clinical trial protocol. The PWG will be independent from project sponsors and free from competing interests.

A project control board (PCB) will be composed of (1) CHF clinical champions, (2) chief investigators, (3) researchers and (4) project managers. The PCB will convene monthly to govern the project and ensure that the project meets all requirements outlined in the project plan. The major responsibilities of the PCB will include (1) reviewing the study plan, (2) providing reports to the project sponsors and ethics committees, (3) deciding budget and administration, (4) resolving contractual issues and (5) maintaining IT and telemonitoring systems in the trial. The PCB will also work with independent management committees for safety and quality of care at corresponding hospitals, to assess the severity of incidents and adverse effects. The PCB will have the capacity to terminate the trial in the event of slow recruitment, safety concerns or overwhelming evidence of benefit.

A Clinical Trial Advisory Committee, composed of chief investigators and research scientists, will convene monthly. The committee will implement and maintain quality assurance and quality control systems to ensure the trial in compliance with the protocol and applicable policies. The committee will also arrange at least one audit during the trial. An arranged audit team will check the overall quality and completeness of the data, examine source documents and ensure that the trial complies with the requirements outlined by the trial protocol, ethics applications and hospital policies. The audit process will be independent from chief investigators and project sponsors.

\section{DISCUSSION}

Daily weight monitoring is a class I recommendation in the management of $\mathrm{CHF}^{11}$ to help maintain fluid balance. Fluid retention is an early sign of acute CHF deterioration, a flag for poor compliance with prescribed medication (especially diuretics) and non-adherence with fluid and salt restrictions. Early identification of abnormal weight fluctuations caused by fluid accumulation allows clinicians to work with the patient to improve their compliance, and provide timely interventions. This potentially reduces the burden of heart failure in terms of reducing preventable hospitalisations and preventing clinical deteriorations among patients with CHF. ${ }^{22} 3839$

In usual care, patients often have difficulties using paper-based diaries to record daily weight entries, and analyse recorded weight entries in association with observed symptoms in order to seek clinical interventions. Weight monitoring in isolation also requires patients to have the knowledge and skills to effectively identify symptoms, make decisions about their clinical relevance and act on these. To overcome these difficulties, this study proposes the ITEC-CHF to automatically detect abnormal weight fluctuations, and provide active clinical support through a call centre and project nurses. Compared with the traditional management of CHF, this programme has the potential to dramatically simplify the daily weight monitoring and management, and does not require special knowledge or skills. While engaging with the patients for interventions of abnormal weight fluctuations, the project nurses have opportunities to assess patients' health, and issues of compliance with other recommendations, such as diet, fluid restriction, medication, exercise and collaborative care. We anticipate that this will allow the nurses to actively engage with patients, and provide a broad range of clinical interventions for the management of CHF. Through the improved compliance and clinical support, it is expected that patients will actively engage with well-established multidisciplinary clinical services for further treatment or care such as titration of diuretic medication.

The efficacy of the programme will be evaluated through a multicentre RCT. The evaluation is focused on the improvement in patient compliance and associated outcomes. To accurately evaluate the compliance with daily weight monitoring, objective weight data will be obtained from both intervention and control groups. A validated questionnaire will also be used to assess participant compliance with other important self-management behaviours, as recommended by CHF guidelines. ${ }^{10}$ In 
addition to compliance, the questionnaire will also assess barriers to self-management of CHF more broadly. This will help in the refinement of telemonitoring to support CHF care in future clinical practice.

If the efficacy is validated, use of the ITEC-CHF will not only improve the patient compliance and health outcomes, but also provide an easy way for care providers to effectively and efficiently engage with patients for collaborative care, especially for patients in rural and remote areas. Based on the ITEC-CHF, other telemonitoring devices such as ambulatory ECG, glucose meters and blood pressure monitors can also be easily integrated to provide broader interventions for patients with CHF who have other cardiac conditions and comorbidities including diabetes and hypertension. Therefore, validation of the system for weight compliance would support further exploration of telemonitoring for improving $\mathrm{CHF}$ care, as well as other chronic conditions.

The study is limited to the 6-month intervention. The 6-month duration is potentially insufficient to reflect real long-term effects of the programme through the ongoing management of CHF. Recent studies, in fact, have already demonstrated an issue of declined patient adherence over time. ${ }^{40}{ }^{41}$ Therefore, extra caution will be exercised when interpreting the outcomes of this study.

\section{Ethics and dissemination}

The ethics application for the trial site in VIC has been approved by Peninsula Health Human Research Ethics Committee (HREC Reference: HREC/14/ $\mathrm{PH} / 27)$, and the ethics applications for Royal Perth Hospital and Fiona Stanley Hospital have been approved by Royal Perth Hospital Human Research Ethics Committee (Reference: 15-081) and the Curtin University Human Research Ethics Committee (Reference: HR 181/2014). The trial has been registered in the Australian New Zealand Clinical Trials Registry (Trial ID: ACTRN12614000916640).

We will report the primary and secondary outcomes regardless of the magnitude and direction of interventional effects or differences between the two trial groups. The report will be disseminated through publication in an appropriate journal, approximately 6 months after finishing data collection.

\section{Author affiliations \\ ${ }^{1}$ The Australian e-Health Research Centre, CSIRO, Brisbane, QLD, Australia \\ ${ }^{2}$ The Australian e-Health Research Centre, CSIRO, Melbourne, VIC, Australia \\ ${ }^{3}$ Allied Health Department and Advanced Heart Failure and Cardiac Transplant \\ Service, Fiona Stanley Hospital, Perth, WA, Australia \\ ${ }^{4}$ School of Physiotherapy and Exercise Science, Curtin University, Perth, WA, Australia \\ ${ }^{5}$ Integrated Care \& Dental, Peninsula Health, Melbourne, VIC, Australia \\ ${ }^{6}$ Department of Cardiology, Peninsula Health, Melbourne, VIC, Australia}

Acknowledgements The authors gratefully acknowledge (1) members of the Project Control Board, including David Anderson, Dr Phil Carrillo, Dr Fergus McGee and Mark Gravell for their management and guidance in the clinical trial, (2) members of MePACS, including Mr Dean Richardson and Mr Dhruv Singh for implementing the telemonitoring system, and managing the call service in the clinical trial, (3) members of Medtech Global, including Mr Rama Kumble and G K
Shridhar for implementing and managing the telemonitoring system in the clinical trial and (4) nursing and medical support provided by Julie Smith and Dr Matthew Best from the trial site in WA.

Contributors This study protocol was mainly developed by HD, AD, IE and RJ. The first draft of the manuscript was written by HD and RJ. All other authors contributed to the design of the clinical study, and/or critical revision of the manuscript.

Funding This project is fully funded by the Health Market Validation Program by Department of State Development, Business and Innovation, Victorian government (121 Exhibition Street, Melbourne, VIC 3001, Australia; the contract was signed in May 2014) and Western Australia Targeted Research Fund Grant by the Department of Health, Western Australia (Notification, 4 February 2014). Primary trial sponsor: Peninsula Health, contact name: IE.

Disclaimer These funding sponsors will not have any roles in the design of this study, execution of the trial, analysis and interpretation of the trial data, and research publications.

Competing interests None declared.

Ethics approval Peninsula Health Human Research Ethics Committee, Royal Perth Hospital Human Research Ethics Committee and the Curtin University Human Research Ethics Committee.

Provenance and peer review Not commissioned; externally peer reviewed.

Open Access This is an Open Access article distributed in accordance with the Creative Commons Attribution Non Commercial (CC BY-NC 4.0) license, which permits others to distribute, remix, adapt, build upon this work non-commercially, and license their derivative works on different terms, provided the original work is properly cited and the use is non-commercial. See: http://creativecommons.org/ licenses/by-nc/4.0/

(c) Article author(s) (or their employer(s) unless otherwise stated in the text of the article) 2017. All rights reserved. No commercial use is permitted unless otherwise expressly granted.

\section{REFERENCES}

1. Ambrosy AP, Fonarow GC, Butler J, et al. The global health and economic burden of hospitalizations for heart failure: lessons learned from hospitalized heart failure registries. J Am Coll Cardiol 2014;63:1123-33.

2. Ponikowski P, Anker SD, AlHabib KF, et al. Heart failure: preventing disease and death worldwide. ESC Heart Fail 2014;1:4-25.

3. Cook C, Cole G, Asaria P, et al. The annual global economic burden of heart failure. Int J Cardiol 2014;171:368-76.

4. Goldberg RJ, Ciampa J, Lessard D, et al. Long-term survival after heart failure: a contemporary population-based perspective. Arch Intern Med 2007;167:490-6.

5. Bui AL, Horwich TB, Fonarow GC. Epidemiology and risk profile of heart failure. Nat Rev Cardiol 2011;8:30-41.

6. Witte KK, Clark AL. Why does chronic heart failure cause breathlessness and fatigue? Prog Cardiovasc Dis 2007;49:366-84.

7. Nieminen MS, Dickstein K, Fonseca C, et al. The patient perspective: Quality of life in advanced heart failure with frequent hospitalisations. Int J Cardiol 2015;191:256-64.

8. Desai AS, Stevenson LW. Rehospitalization for heart failure: predict or prevent? Circulation 2012;126:501-6.

9. Butler J, Kalogeropoulos A. Worsening heart failure hospitalization epidemic we do not know how to prevent and we do not know how to treat!. J Am Coll Cardiol 2008;52:435-7.

10. National Heart Foundation of Australia and the Cardiac Society of Australia and New Zealand (Chronic Heart Failure Guidelines Expert Writing Panel). Guidelines for the prevention, detection and management of chronic heart failure in Australia. (Epub ahead of print Oct 2011).

11. Ponikowski P, Voors AA, Anker SD, et al. 2016 ESC Guidelines for the Diagnosis and Treatment of Acute and Chronic Heart Failure. Rev Esp Cardiol 2016;69:1167.

12. Morrissey RP, Czer L, Shah PK. Chronic heart failure: current evidence, challenges to therapy, and future directions. Am J Cardiovasc Drugs 2011;11:153-71.

13. Wright SP, Walsh $\mathrm{H}$, Ingley KM, et al. Uptake of self-management strategies in a heart failure management programme. Eur J Heart Fail 2003;5:371-80.

14. van der Wal MH, van Veldhuisen DJ, Veeger NJ, et al. Compliance with non-pharmacological recommendations and outcome in heart failure patients. Eur Heart J 2010;31:1486-93. 
15. van der Wal MH, Jaarsma T, van Veldhuisen DJ. Non-compliance in patients with heart failure; how can we manage it? Eur $J$ Heart Fail 2005;7:5-17.

16. van der Wal MH, Jaarsma T, Moser DK, et al. Compliance in heart failure patients: the importance of knowledge and beliefs. Eur Heart $J$ 2006;27:434-40.

17. Jeon $\mathrm{YH}$, Kraus SG, Jowsey $\mathrm{T}$, et al. The experience of living with chronic heart failure: a narrative review of qualitative studies. $B M C$ Health Serv Res 2010;10:9.

18. Ni H, Nauman D, Burgess D, et al. Factors influencing knowledge of and adherence to self-care among patients with heart failure. Arch Intern Med 1999;159:1613-9.

19. Toh CT, Jackson B, Gascard DJ, et al. Barriers to medication adherence in chronic heart failure patients during home visits. Journal of Pharmacy Practice and Research 2010;40:27-30.

20. Ruppar TM, Cooper PS, Mehr DR, et al. Medication adherence interventions improve heart failure mortality and readmission rates: systematic review and meta-analysis of controlled trials. J Am Heart Assoc 2016;5:e002606.

21. Eastwood CA, Travis L, Morgenstern TT, et al. Weight and symptom diary for self-monitoring in heart failure clinic patients. $J$ Cardiovasc Nurs 2007;22:382-9.

22. Jones CD, Holmes GM, Dewalt DA, et al. Is adherence to weight monitoring or weight-based diuretic self-adjustment associated with fewer heart failure-related emergency department visits or hospitalizations? J Card Fail 2012;18:576-84.

23. Bashi N, Karunanithi M, Fatehi F, et al. Remote monitoring of patients with heart failure: an overview of systematic reviews. J Med Internet Res 2017;19:e18-93.

24. Kitsiou S, Paré G, Jaana M. Effects of home telemonitoring interventions on patients with chronic heart failure: an overview of systematic reviews. J Med Internet Res 2015;17:e63.

25. Hasan A, Paul V. Telemonitoring in chronic heart failure. Eur Heart $J$ 2011;32:1457-64.

26. Ramaekers BL, Janssen-Boyne JJ, Gorgels AP, et al. Adherence among telemonitored patients with heart failure to pharmacological and nonpharmacological recommendations. Telemed J E Health 2009;15:517-24.
27. Seto E, Leonard KJ, Cafazzo JA, et al. Mobile phone-based telemonitoring for heart failure management: a randomized controlled trial. J Med Internet Res 2012;14:e31.

28. Hale TM, Jethwani K, Kandola MS, et al. A remote medication monitoring system for chronic heart failure patients to reduce readmissions: a two-arm randomized pilot study. J Med Internet Res 2016;18:e91.

29. Vuorinen AL, Leppänen J, Kaijanranta $\mathrm{H}$, et al. Use of home telemonitoring to support multidisciplinary care of heart failure patients in Finland: randomized controlled trial. J Med Internet Res 2014;16:e282-9.

30. Doig GS, Simpson F. Randomization and allocation concealment: a practical guide for researchers. J Crit Care 2005;20:187-91.

31. MePACS. https://mepacs.com.au/ (accessed 12 Mar 2017).

32. Evangelista LS, Berg J, Dracup K. Relationship between psychosocial variables and compliance in patients with heart failure. Heart Lung 2001;30:294-301.

33. https://euroqol.org/ (accessed 20 Jun 2017).

34. Ding H. Six Minute Walk Test (6MWT) instructions. http://www. heartonline.org.au/media/DRL/6MWT_standardised_instructions.pdf (accessed 20 Jun 2017).

35. Hare DL, Selvadurai LP, Wang JQ, et al. Psychometric assessment of the Cardiac Depression Scale Short Form in cardiac outpatients. Eur J Cardiovasc Nurs 2017;16:249-55.

36. Ding H. Clinical frailty scale. $2008 \mathrm{http} / / / \mathrm{www} . c a m a p c a n a d a . c a /$ Frailtyscale.pdf (accessed 20 Jun 2017).

37. Wittes J. Sample size calculations for randomized controlled trials. Epidemiol Rev 2002;24:39-53.

38. Tsuyuki RT, McKelvie RS, Arnold JM, et al. Acute precipitants of congestive heart failure exacerbations. Arch Intern Med 2001;161:2337-42.

39. Jones CD, Holmes GM, DeWalt DA, et al. Self-reported recall and daily diary-recorded measures of weight monitoring adherence: associations with heart failure-related hospitalization. BMC Cardiovasc Disord 2014;14:8.

40. Stut W, Deighan C, Cleland JG, et al. Adherence to self-care in patients with heart failure in the HeartCycle study. Patient Prefer Adherence 2015;9:1195-206.

41. Chaudhry SI, Mattera JA, Curtis JP, et al. Telemonitoring in patients with heart failure. N Engl J Med 2010;363:2301-9. 